\title{
Unifying and Targeting Cultural Activities via Events Modelling and Profiling
}

\author{
Erik Mannens \\ Ghent University - IBBT \\ ELIS - Multimedia Lab \\ 9050 Ghent, Belgium \\ erik.mannens@ugent.be \\ Kristof Geebelen \\ K.U. Leuven - IBBT \\ Distrinet \\ 3001 Leuven, Belgium \\ kristof.geebelen@cs.kuleuven.be
}

\author{
Sam Coppens \\ Ghent University - IBBT \\ ELIS - Multimedia Lab \\ 9050 Ghent, Belgium \\ sam.coppens@ugent.be \\ Hendrik Dacquin \\ VRT \\ VRT-medialab \\ 1043 Brussels, Belgium \\ hendrik.dacquin@vrt.be
}

\author{
Toon De Pessemier \\ Ghent University - IBBT \\ INTEC - WiCa \\ 9050 Ghent, Belgium \\ tdpessem@intec.ugent.be \\ Rik Van de Walle \\ Ghent University - IBBT \\ ELIS - Multimedia Lab \\ 9050 Ghent, Belgium \\ rik.vandewalle@ugent.be
}

\begin{abstract}
Today, people have a lot of spare time at their hands which they want to fill in according to their interests, whereas cultural temples are trying to attract interested communities to their carefully planned cultural programs. These cultural activities can be characterised as dynamic and distributed events in addition to which it is important to aggregate, enrich, recommend, and distribute these event items as targeted as possible. In this paper, we show how personalised recommendation and distribution of events, described using an RDF/OWL representation of the EventsML-G2 standard, can be enabled by automatically categorising and enriching events metadata via smart indexing and open linked datasets available on the web of data. As such, the ultimate goal of the CUPID-project is to provide an open, userfriendly platform that harnesses the end-user with a tool to access useful event information that goes beyond basic information retrieval. At the same time, we provide the (inter)national cultural community with standardised mechanisms to describe/distribute event and profile information.
\end{abstract}

\section{Categories and Subject Descriptors}

H.3.3 [Information Storage and Retrieval]: Information Search and Retrieval-clustering, information filtering, relevance feedback

\section{General Terms}

Design, Standardisation

\section{Keywords \\ Event Modelling, Profiling, Recommendation}

\footnotetext{
Permission to make digital or hard copies of all or part of this work for personal or classroom use is granted without fee provided that copies are not made or distributed for profit or commercial advantage and that copies bear this notice and the full citation on the first page. To copy otherwise, to republish, to post on servers or to redistribute to lists, requires prior specific permission and/or a fee.

EiMM'09, October 23, 2009, Beijing, China.

Copyright 2009 ACM 978-1-60558-754-7/09/10 ...\$10.00.
}

\section{INTRODUCTION}

The nature of information has changed significantly in the last two decades. Now information is multimedia on the internet, sensitive to its spatio-temporal roots, live, and dynamic. This also holds true for the cultural scene where supply and demand has shifted from hard-copy press to the internet as well. As such an important role is granted to cultural temples to grasp the vast and complex supply of cultural and leisure events [6]. Cultural organisations are no longer only traditional gate keepers who determine what the public needs to see, but their primary function is shifting to being a guide that puts emphasis and structure on the supply and demand of these cultural events and their accompanying assets. We believe that to deal with such dynamic distributed information, events should be used as the fundamental basis in organising and accessing cultural activities information. The event model used in our approach captures the semantics involved in supporting recommendation of cross-genre cultural activities. Our ideas are demonstrated in the context of an end-to-end events distribution platform CUPID ${ }^{1}$ that is being implemented for the Flemish cultural scene using multiple aggregated and enriched information sources.

This paper is organised as follows. In Section 2, we present the EventsML-G2 $2^{2}$ standard and its conceptualisation in an $\mathrm{OWL}^{3}$ ontology as a unifying (meta)datamodel. Section 3 highlights the workflow-based bus-infrastructure to be used as a back-end system for the continuous flow of cultural events. Section 4 further elaborates on how the different flow of events can be aggregated and clustered, whereas Section 5 shows how these events and their accompanying multimedia can be automatically enriched with knowledge available in large linked datasets. Afterwards Section 6 and Section 7 unleash the harvested user profiles to the recommendation engine to harness the best-fit cross-genre cultural activities. Section 8 then distributes these preferred and enriched cultural activities to the individual users. Finally, conclusions are drawn in Section 9.

\footnotetext{
$1_{\text {https://projects.ibbt.be/cupid }}$

2 http://www.iptc.com/std/EventsML-G2/EventsML-G2_1.1.zip

3 http://www.w3.org/TR/owl-ref/
} 


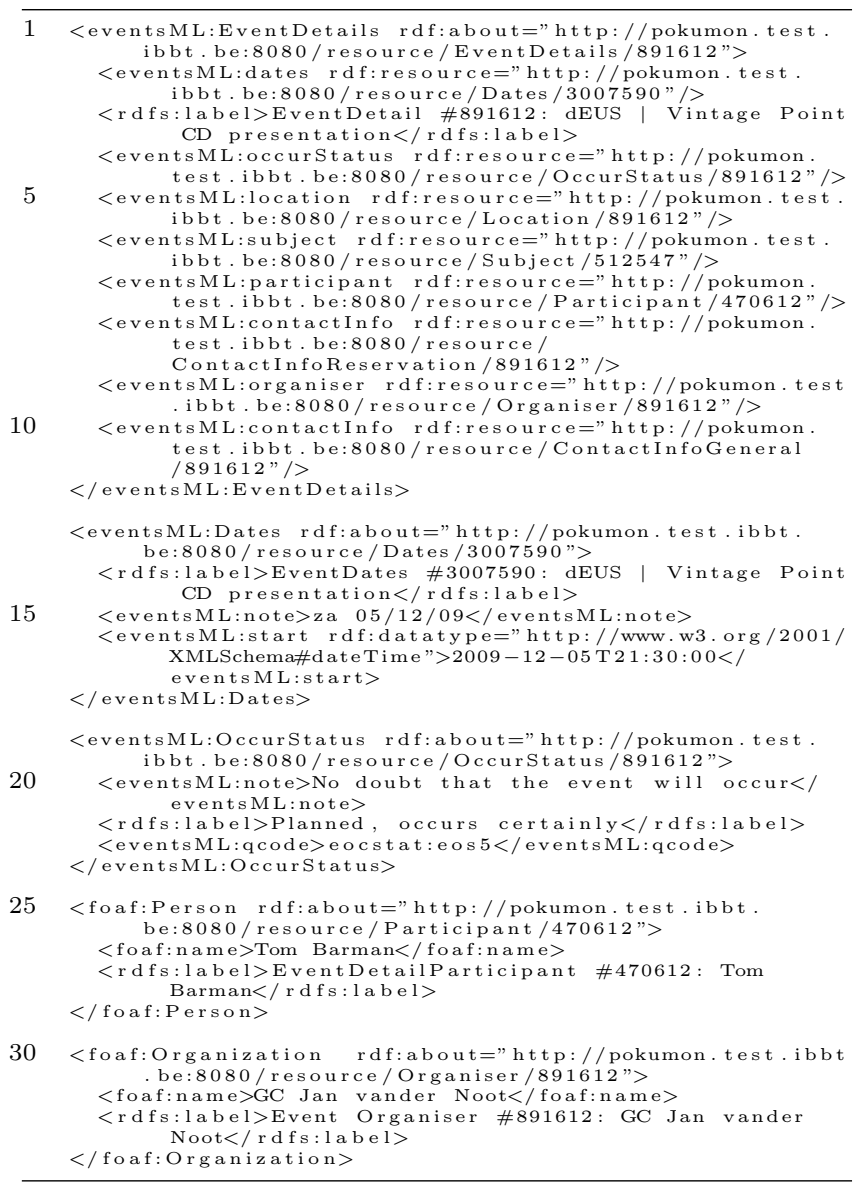

Listing 1: RDF sub-example of EventsML-G2

\section{EVENT MODELLING}

In Flanders, CultuurNet ${ }^{4}$ acts as an aggregator for all the (local) cultural events. They already have a platform which allows event organisers to upload their events in a proprietary way. Furthermore, these events are stored using a proprietary XML schema ${ }^{5}$ and dissemination occurs for each customer in an ad hoc manner. Within the CUPID-project we developed a semantic metadata model that can be used in a standardised way both by the supply and demand side. Using a semantic metadata model has many advantages: it allows linking information from multiple sources, e.g., we can provide information on a play, combined with information on the writer of the play, or hotels in the neighbourhood of the theatre.

It empowers also other data providers to easily use your data, e.g., it lets us distribute the most valuable information to the end users via a recommendation system, as will be elaborated on over the next few Sections. Another advantage of a semantic metadata model is its reasoning capabilities, which enables answering complex queries, e.g., filter all theatre events that play in a theatre in a certain area, that take place during the weekend, and perform a play from a certain writer.

\footnotetext{
$4_{\text {http: //www. cultuurnet.be }}$

http://www.w3.org/TR/2008/REC-xml-20081126/
}

The standard we used for the modelling and dissemination of the event records is EventsML-G2. This is an International Press Telecommunications Council ${ }^{6}$ (IPTC) standard for conveying event information in a news industry environment. IPTC is responsible for many news exchange formats, a.o. NewsML-G2 ${ }^{7}$ and SportsML-G2 ${ }^{8}$, which are languages (together with EventsML-G2) that are built on top of the IPCT News Architecture framework (NAR). NAR is a generic model that defines four main objects (newsItem, packageItem, conceptItem and knowledgeItem) and the processing model associated with these concepts. Apart from these news exchange formats, IPTC is also responsible for maintaining the controlled vocabularies of NewsCodes ${ }^{9}$. A NewsCode is a single code for representing a concept, which is used for categorisation. These NewsCodes come from the News Architecture framework. They are used to assert something about the content of a news item. This could be done using free text or codes. The advantage of NewsCodes is that they can be easily shared, that they require a definition allowing not only to share the NewsCode, but also the semantics of the NewsCode, and that they are language agnostic. NewsCodes can be grouped into four categories: Descriptive NewsCodes to describe the content of the news items, e.g., subj:01000000, which indicates that the subject of an object is arts, culture and entertainment; Administrative NewsCodes for proper administration of news items, e.g., pst1:Usable, which indicates that the content may be published without any restriction; Transmission NewsCodes for describing the transmission process, e.g., prio:1 that indicates that the content has highest priority; and Exchange Format NewsCodes to support specific functionalities of the different IPTC news standards, amongst them EventsML-G2, e.g., eocstat:eos5, which is a EventsML-G2 specific NewsCode that states that the event is planned and will certainly occur.

EventsML-G2 is intended for receiving and storing information about an event from the event organiser, and for publishing information about a specific event by a (news) provider. It allows describing the events in different languages and allows interlinking the events, e.g., indicating that a certain event is part of a bigger event. Currently, the standard is described as an XML schema. In order to make it usable in the Semantic Web Stack, we developed an OWL ontology of that standard. Semantic models can be described in various ways, e.g., $\mathrm{RDFS}^{10}$ or OWL. OWL is a richer language to construct models, e.g., it allows putting cardinality constraints on properties, and OWL permits to mix RDFS and OWL constructs. As a consequence, the semantic model of the EventsML-G2 standard can be described more precisely using the OWL language. The EventsML-G2 standard has two ways for representing the persisting knowledge facet of event information: as conceptItem or as knowledgeItem. A conceptItem aims to convey knowledge about a unique concept, i.e., event. A knowledgeItem bundles a set of concept components, which are managed as a whole. In the context of publishing events from event organisers, events are modelled in our ontology as conceptItems. Each conceptItem describes just one event.

\footnotetext{
$6_{\text {http: //www.iptc.org }}$

7 http://www.iptc.com/std/NewsML-G2/NewsML-G2_2.2.zip

8 http://www.iptc.com/std/SportsML/2.0.zip

10 http://www.iptc.org/cms/site/index.html?channel=CH0088

10 http://www.w3.org/TR/rdf-schema
} 
Our ontology follows the specifications of the EventsMLG2 standard. The main difference with the specification is that agents (persons or organisations) are described using the $\mathrm{FOAF}^{11}$ ontology. Following the specifications has the advantage of describing events in a language agnostic way, using the NewsCodes. Yet, the FOAF ontology was chosen to describe the agents, because it is broadly used for descriptions about persons or organisations. This allows to easily incorporate richer FOAF descriptions from other datasets into the events descriptions. Our developed ontology (partly shown in Listing 1) describes events by a root class conceptItem. This class can be seen as a 'wrapper' class, which bundles administrative metadata with descriptive metadata of the event. For this, the conceptItem class has three object properties which relate the conceptItem class to one itemMeta class and one contentMeta class, which provide the administrative metadata, and at least one concept class, which describes the event. Furthermore, the conceptItem class has also some datatype properties, e.g., catalogRef which indicates the schema used for describing the events as concept items. The itemMeta class gives, a.o., the publication status of the event, the provider of the event, and the class of the event, indicating how the event was described, i.e., as a conceptItem. For the publication status of the event and its class, NewsCodes are used, e.g., stat:usable for the publication status, indicating the event can be published and cinat:concept for the class, which stipulates that the event is described as a concept and not a knowledge item. The contentMeta class tells when the record was created and modified. The concept class holds the actual information about the event. It uses five object properties:

- eventDetails: relating the concept class to the eventDetails class.

- broader, narrower, related, and sameAs: owl:sameAs for indicating that two concepts describe the same event, skos:broader, skos:narrower, and skos:related for pinpointing the relations between events. For instance, a festival can consist of different concerts. The concert descriptions are related to each other via skos:related, the concert records are related to the festival record using skos:broader, and, vice versa, the festival description is related to the concert descriptions through the skos:narrower relation.

The concept class has also some datatype properties, e.g., name, a label for the event; type, indicating that the concept describes an event; or note, which gives a description of the event. The type of the concept is indicated by a NewsCode, which is for events cpnat:event. The concept class has also a datatype property indicating the language the EventsMLG2 description was made up. The eventDetails class gives a more detailed description of the event. For this, the class has eleven object properties linking the eventDetails class to other classes:

- dates: relates eventDetails to the dates class, which holds the start and end date/time of the event, or the duration of the event. The start and end date/time can be described as a point of date/time or as date/time interval. Furthermore, the dates class can also contain some recurrence information about the event.

\footnotetext{
$11_{\text {http://xmlns.com/foaf/spec/ }}$
}

The recurrence of an event is expressed as a list of dates or a recurrence rule.

- location: referring to the location class, which holds information about the location where the event takes place. This location information can be expressed by its geospatial coordinates and by its contact information which is expressed by the contactInfo class (see hereafter).

- participant: links the event to a FOAF person or organisation class, describing persons or organisations participating in the event.

- organiser: relates also to a FOAF person or organisation class, indicating the organiser of the event.

- contactInfo: refers to the contactInfo class, which denotes the contact information for the event, e.g., email addresses, addresses, web URLs, phone numbers, and fax numbers. This class also has a datatype property for specifying the role of the contact information. The role of the contact information is also expressed by a NewsCode, e.g., ecirol:general for general contact information, and ecirol:ticketing for the contact information for reserving tickets to the event.

- language: relates the eventDetails class to the Language class, which gives information of the languages used in the event. This class gives the name of the language and its role, expressed by a NewsCode, e.g., lrol:subtitle

- subject: refers to the subject class, which gives the NewsCodes of the subjects of the event and a literal description of that NewsCode.

- occurStatus: links the event to the occurStatus class. This class expresses the certainty of the occurrence of the event by a NewsCode, e.g., eocstat:eos5, and a literal description of that NewsCode, which says that eocstat:eos 5 means that the event is planned and certainly occurs.

- registration: refers to the registration class, which gives extra registration information of the event in the form of a literal description, e.g., the price information.

- participationRequirement: links the eventDetails class to the participationRequirement class. This class gives some information about the participation requirements for the event, e.g., the minimum age for attending the event.

- media: referring to the media class, which holds references to certain media files, belonging to the description of the event, e.g., a promotion movie.

The current ontology is published on Multimedia Lab's website $^{12}$. By providing a semantic version of (for the moment) part of the IPTC standard EventsML-G2, our ontology acts as a unifying layer, relating all the information coming from the different data providers, and allowing the events to be queried using a SPARQL ${ }^{13}$ endpoint.

\footnotetext{
12 http://multimedialab. elis. ugent.be/ontologies/EventsML-G2/v1.0

13 http://www.w3.org/TR/rdf-sparql-query/
} 


\section{GENERAL ARCHITECTURE}

Our current architecture that supports the flow of personalised, recommended cultural events gives an overview of the integration layer that connects the different components that realise the proposed requirements through the above proposed semantic events (meta)datamodel. The infrastructure is a loosely coupled bus-architecture consisting of $6 \mathrm{com}-$ ponents: aggregation, categorisation, enrichment, profiling, recommendation, and distribution. All 6 will be elaborated on in the next Sections, but a general overview highlighting the overall architectural flow is given here. The flow starts with content aggregation where cultural data is retrieved from different information sources. Different strategies for content retrieval are possible. It can be actively searched on the web (pull) or content can be sent by partners or individual end-users (push). The basic information from the aggregation is the main ingredient for the second phase where categorisation is done. A taxonomy builder classifies events according to themes. Adding intelligent information to the event data helps to find more accurate information in later phases, i.e. during content enrichment. Enrichment means adding extra useful information, a.o. cross-referenced multimedia, to the basic event info that helps end-users in planning their activities. Based on the properties of the event, its ontology can be complemented with information of other sources. For example, an event concerning a theatre play on Shakespeare can be enriched with related literature information from libraries or footage of a former, relevant play of the same theatre company. Also information on neighbouring hotels can be added from a tourist service, based on the location of the event. Another important requirement is to provide the end-user with accurate information. By means of a user-based profile, the infrastructure can look for alternative recommendations to the initial search operation. By using profiles and a recommendation engine, we aim to provide the end-user with accurate and personalised event information, complemented with interesting recommendations. In the final phase, the results are sent to the customer. The distribution layer provides on-line publication channels (e.g. websites) with access to the different data sources. Events can be searched by individuals or event information can be used and published by other organisations. The following sections discuss these different phases in more detail.

For the implementation of the integration layer, we use a Service Oriented Architecture ${ }^{14}$ (SOA) as the underlying state of the art technology. An Enterprise Service Bus (ESB) provides a means of consolidating, connecting and organising business services into a new cohesive solution. The requirement categories discussed above can be modelled as separate services where the SOA infrastructure allows them to exchange data with one another as they participate in business processes. The aim is to have a loose coupling between the different modules of the system. Services can be very heterogeneous. Their implementation is language and technology independent; only communications must be agreed on. The high flexibility supported by this technology allows an easy integration of new sources (i.e. aggregation sources or distribution targets) and remove or modify existing sources without interrupting the working system. Using a standardised technology has also the advantage of reusable

$14_{\text {http://opengroup.org/projects/soa/ }}$ existing middleware services like security, logging, etc. Orchestration between services is handled by a workflow engine. This engine is integrated in the service bus architecture and supports the execution of WS-BPEL processes. Business Process Execution Language for Web Services ${ }^{15}$ (WSBPEL) provides a language for the specification of business processes and business interaction protocols. WS-BPEL is built on top of XML specifications. For example, it uses the web service description language ${ }^{16}$ (WSDL) to describe the web service interfaces that participate in a process. An executable WS-BPEL process is defined by a control flow that consists of a combination of basic and structured activities. Communication in the aggregation layer involves mainly exchanging (meta)data related to the cultural events. The events data, modelled via our events ontology, can thus easily be described in RDF/XML ${ }^{17}$. WS-BPEL (and SOA in general) supports the 'Simple Object Access Protocol' (SOAP). SOAP is a protocol specification for exchanging structured information between services. Since it relies on XML as its message format, it is a natural choice as communication protocol.

\section{AGGREGATION \& CATEGORISATION}

\subsection{Aggregation}

Aggregation concerns the retrieval of basic information of cultural events from different data sources. Some sources need to be authenticated and their input data needs to be validated. Since the input sources use different data formats, a transformation to our standard event ontology is executed. The feeds need to be aggregated periodically. Therefore we use a scheduler that triggers the aggregation process for specific feeds. During aggregation content indexing runs through the individual objects in a feed. In a first step the object will be enriched with geographical coordinates if they are not already available. Objects without these coordinates will not be added to the index. Double detection (an object with the same date and the same geographical coordinates will not be added to the index) eliminates redundant data. During the aggregation flow, there is interaction with other services like the taxonomy builder of the categorisation module, and the enrichment modules. Finally the import flow of data ends by storing the results in an RDF-triplestore.

\subsection{Categorisation}

Before enrichment, the data needs to be categorised first to allow a targeted search for related information. Here comes the categorisation module into play. In contrast to other indexing technologies, i.Know's Information Forensics - Smart Indexing ${ }^{18}$ is based on a bottom-up approach. i.Know thus approaches indexation and analysis of documents in a totally different manner than other technologies. Instead of the top-down approach, retrieving only the information pre-defined in an index or taxonomy, Smart Indexing starts with the document itself and the possible information it contains. Instead of generating indexation lists by means of single keyword lists, it identifies all meaningful word groups in the non-structured information stream of

\footnotetext{
15 http://docs. oasis-open. org/wsbpel/

16 http://www.w3.org/TR/2007/REC-wsd120-20070626/

17 http://www.w3.org/TR/rdf-syntax-grammar/

18 http://www.iknow.be
} 
events data. The benefits of this methodology are clear: a) Efficiency: no generation of meaningless word combinations; b) High Precision: all information is to be retrieved. This way, a total retrieval of all available information is realised without intensive training procedures for the analysing program. Top-down solutions are often based on intensively construed taxonomies and/or ontologies, attempting to list all available information. Non-listed words and concepts, synonyms and spelling variants disappear in the haystack. On the other hand, CUPID's Smart Indexing yields more and more reliable results. Our taxonomy builder thus classifies events according to common found themes. These themes can then be linked to related info.

\section{ENRICHMENT}

As the EventsML-G2 ontology has been modelled, the conversion of the (meta)data of individual event streams into RDF according to this ontology is straightforward as was described in Section 2 and Section 4, e.g., converting events from CultuurNet, described by a proprietary XML schema, i.e. $\mathrm{CDBXML}^{19}$, to our EventsML descriptions. However, we advocate a further step aiming at enriching the events (meta)data semantically at intake following the linked data principle ${ }^{20}$. In our case, we apply linguistic processing on the plain text contained into the $4 \mathrm{~W}$-elements (what, where, who, when) of the metadata and the main description of the events.

The linguistic processing consists in extracting named entities such as persons, organisations, companies, brands, locations and other events. We use both the i.Know's Information Forensics service and the OpenCalais infrastructure ${ }^{21}$ for extracting these named entities. For example, the processing of the headline "Tom Barman and his band dEUS opening their latest album Vantage Point in Rock Werchter" will result in five named entities: 'Tom Barman','dEUS', 'Vantage Point', 'Rock Werchter', and 'Werchter' together with their type (i.e. Person, Music Group, Music Album, Event, Location, etc.). Once the named entities have been extracted, we map them to formalised knowledge on the web available in GeoNames ${ }^{22}$ for the locations, or in DBPedia ${ }^{23}$ for the persons, organisations and events. The string 'Tom Barman' is therefore mapped to its URI in DBPedia ${ }^{24}$ that provides $i$ ) a unique identifier for the resource and ii) formalised knowledge about this person such as his biography, career and genealogy in multiple languages. Therefore, the use of the OpenCalais web service allows us to populate the knowledge base by providing a list of possible instances for all named entities discovered.

The main challenge in this semantic enrichment step is then to deal with the ambiguity. For example, the GeoNames web service tends to return all cities over the world when a single string is passed as an argument, and no country is specified. Fortunately, most events contain always information about the city and the country yielding accurate recognition of the location mentioned in the event. When the accuracy is the primary concern, we envision a semiautomatic approach where suggestions will be proposed to

\footnotetext{
19 http://www. cultuurdatabank. com/XMLSChema/CdbXSD/3.0/FINAL/CdbXSD. xsd

20 http://linkeddata.org/

21 http://www. opencalais. com/

22 http://www.geonames.org

23 http://dbpedia.org

$24_{\text {http://dbpedia.org/resource/Tom_Barman }}$
}

the editors during the annotation process. Furthermore we integrated the extracted metadata from $\mathrm{BibNet}^{25}$ (dissemination of all bibliographic information of Flemish public libraries on authors, which are described as FOAF persons) and Toerisme Vlaanderen ${ }^{26}$ (dissemination of all touristic information of Flemish cities and regions, which are described using a proprietary OWL ontology) to be able to also further enrich these extracted named entities and link them to other A/V-media types using the available data from $V R T^{27}$ (dissemination of cultural multimedia assets which are described using the related NewsML-G2 standard).

\section{PROFILING}

The empowerment of the user is a phenomenon of the recent years. The user wants personalised information, based on his profile. The same holds for events. Nowadays, there is an enormous offer of events. Most events are irrelevant for a particular user, because the event is not interesting for the user, or the event is too far away. Recommendation systems already try to offer content tailored to the user's needs. An example of this is pushing information, linked to the user's interests, based on the past events the user attended.

Together with the evolution of Web2.0, social networks became very popular. Today, many users have several profiles in various social networks. Even many cultural temples/event organisers, e.g., Vooruit ${ }^{28}$, have their own social network where users can make up a personal profile and befriend other users. This profile is a mix of static information (e.g. the user's all-time favourite movies, band and books), dynamic information in the form of an activity stream (e.g. what the user is listening to or what the user is up to), and the user's social graph (the social connections with other users). All this profile information is very useful to a recommendation engine for offering personalised information.

Current recommendations are offered within the closed context of a single community: e.g. Facebook ${ }^{29}$ recommends events based on RSVP event invitations ${ }^{30}$ from other users connected to the Facebook user's social graph. Facebook does not automatically recommend events based on the static and dynamic profile information of a user, nor does it take into account possibly interesting data coming from other social networks. Another issue for event organisers is that the events they publish are not automatically aggregated and transformed into a Facebook event. Even more, if an event organised by the Vooruit is available in Facebook, RSVP responses are not propagated back to the event organiser, hence important feedback is lost that is useful to both the event organiser and the end user (e.g. it could fine-tune event recommendations to that user). This set the premise to develop a Facebook service and a Facebook application as can be seen in Figure 1:

- A Facebook eventLogger service: eventLogger logs all events that are maintained by event aggregator Cultuurnet and transforms them into Facebook events. Thus all events are automatically transformed and synchronised into Facebook events, eliminating the need

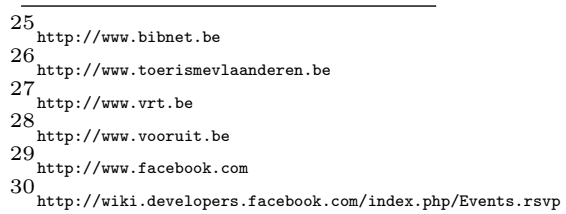




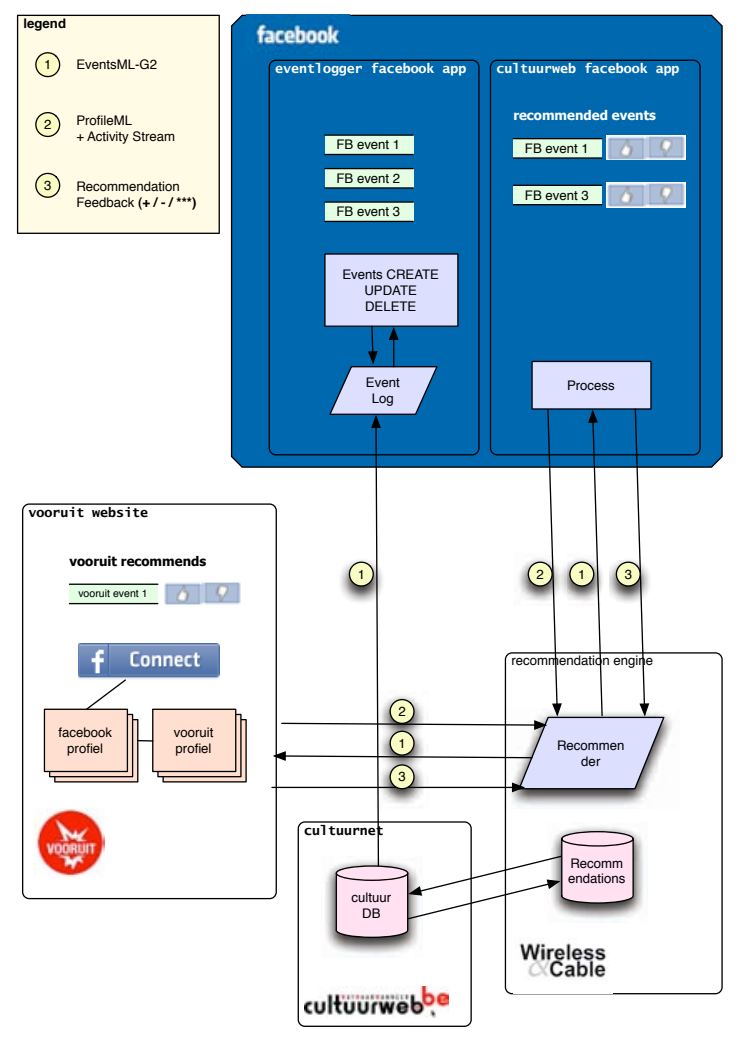

Figure 1: Profiling Flow

for cultural organisers to repeat the routine of inputting events in several web event aggregators (Facebook and Cultuurnet) and thus also eliminating the cumbersome task of making changes to events, should they happen afterwards. This logging service could be easily extended in order to include other cultural event aggregators on the web (last.fm, myspace, etc.). This eventLogger receives events in EventsML-G2 format from the Flemish cultural event aggregator's database and outputs the events in Facebook's native event format as documented in the Facebook API. Current limitations include the inability to add images (e.g. an event poster) as this is not yet implemented in the Facebook API.

- A Facebook eventRecommender application: eventRecommender presents Facebook events generated by the eventLogger to the user based on the user's Facebook profile.

The information fields, stored in the profile for calculating the recommendations, are: a) User specific event information: RSVP information of past and future events; b) Static Profile: Favourite music, movies, etc.; and c) Dynamic Profile: Location of the user, etc. Within the application the user can provide four types of recommendation feedback: 'Attending', 'Maybe Attending', 'Not Attending, but this is a good recommendation', and 'Not Attending, because it is a bad recommendation'. This feedback propagates back to our recommendation engine to adjust the recommendations.
However one doesn't want to limit the recommendations based solely on the Facebook profile. Other social networks have interesting information about a user that could have a detrimental effect on a good recommendation (e.g. the ticketing information stored by the Vooruit). When a user buys a ticket for a Vooruit event via the Vooruit website, this ticketing information becomes part of his Vooruit profile, and not of his Facebook profile. Therefore, we developed a global profile, which can be filled with information coming from different user profiles. This new global profile can then be used by the recommendation engine to calculate the recommendations. By providing a Facebook connect for instance on the Vooruit Website, the users from the Vooruit user community can be uniquely linked to their Facebook profile. The choice for Facebook as a central hub for connecting the different user profiles, is justified by the fact that Facebook is currently the most popular user community. Based on this information, the recommendation engine can start calculating recommendations based on this global profile, which holds information from many profiles the user has in different user communities.

\section{RECOMMENDATION}

The Internet as a new communication model for event information stimulates the announcement and propagation of events to the general public. New events become acquainted for a big mass of people who could never have been reached in the past. The offer of available events becomes more detailed, more extensive and by the introduction of niche events, also more varied. However, this enormous offer of events and its continuous growth makes the selection process increasingly difficult for end-users who can only attend a small fraction of these events anyway. Furthermore, the majority of the events are irrelevant, not in the field of interest of the user or do not match personal requirements (e.g. price, location). Many web sites use a keyword based search tool to help the consumer with finding specific content. Nevertheless, this rudimentary search tool is not capable to weed out irrelevant content or to take personal preferences into account. Recommendation systems try to solve this problem by creating and updating a user profile in the background and then filtering and recommending content according to the gathered preference information.

The overabundance of content and the related difficulty to discover interesting content items is already addressed in several contexts. Online shops, like Amazon, apply collaborative filtering to personalise the online store according to the needs of each customer [5]. The items that customers purchase or explicitly rate will represent their interests and are used for future recommendations [3]. Netflix ${ }^{31}$ is an online, mail-based, DVD rental service to customers in the United States. After renting a movie, customers may assign a rating to the movie, using a concrete score from 1 to 5 , which builds up their personal movie profile. Not only for online content, but also on the digital TV platform are recommendation techniques intensively applied. Several personalised TV guide systems which filter and recommend TV programs according to the user's preferences, are already developed for set-top boxes [9] and personal digital recorders $[4,8]$. Because of the success of recommendation techniques for a big variety of items (books, DVDs, TV programs), it

$31_{\text {http://www.netflix.com }}$ 
sounds logically to use the same recommendation techniques for suggesting events. However some problems arise due to the inherent nature of events [2].

Most existing recommender systems employ collaborative filtering techniques to make predictions about which item a user is likely to be interested in. These kinds of techniques identify correlations between users and recommend items which similar users have liked in the past. To determine these correlations, a variety of information sources are utilised, ranging from past purchase records, explicit ratings or comments on items/events, to implicit click or viewing behaviour. However, these traditional collaborative filtering techniques have difficulties with sparse data sets, which result in inaccurate recommendations. Especially event systems suffer from this sparsity problem, since most users only attend a small fraction of all the available events. Grouping people or items/events into clusters can be a solution, but finding the optimal clusters is a tricky task [7].

Moreover, collaborative filtering systems cannot cope at all with time-specific items such as events, which typically receive their consumptions (i.e. ratings, comments, etc.) after they have finished. So, for the majority of events that exist as a one-time occurrence, the recommendations are already meaningless as soon as they can be calculated. Consequently, generating event recommendations is a special case in which we have to decide whether to recommend items which have not yet been rated or commented at all. Content based algorithms, which consider the meta information of items and recommend items similar to those a user liked in the past can partly make up for that drawback, but the collaborative feature is totally lost on them.

To overcome these problems we opted for a hybrid collaborative filtering - content based technique whose rationale is concisely summed up as "recommending future items if they are similar to the past ones that similar users have liked". This approach has proven its efficiency for recommending events in the past [2]. Before the algorithm is capable to generate personal recommendations, two different similarity measures have to be calculated based on the available data. In the first phase, user similarities have to be calculated for each couple of end-users, based on their past consumption behaviour. For this similarity measure, standard calculation metrics as used in traditional collaborative filtering systems (e.g. cosine similarity, mutual information or Pearson correlation), will be applied. In the second phase, item similarities will determine how related two events are. To calculate the similarity between a past event, which is already evaluated by the users, and a future event, which is a candidate recommendation, the system uses the semantic metadata model of the events as described in Section 2. The object properties of the eventDetails class constitute the input for the similarity metric. To extend the data set, which originally consists of user evaluations for past events, with evaluations for future events, the end-users have the possibility to check the "I want to see this event" option in the user interface. This positive rating mechanism for future events, together with the metadata based similarity metric, will help the algorithm to generate similarities between past and future events.

In order to take into account personal event selection criteria that are not related to the subject of the event, we added some contextual post-filters to the recommendation system. These filters will operate after the main recommendation algorithm and remove or penalise the candidate recommendations which do not satisfy the personal selection criteria. These personal selection criteria, which can be specified by the end-user, are for example the price, the language, the participation requirements, the location, and the date of the event.

\section{DISTRIBUTION}

\subsection{Publishing events as Linked Open Data}

Now that we have the enriched events described in our events ontology, we can start publishing the events as Linked Open Data (LOD), which lets people share structured data on the web as easily as they share documents today. LOD refers to a style of publishing and interlinking structured data on the web and lets you use RDF data models to publish the structured data on the web and uses RDF links to interlink data from different datasets. This makes the web one giant database, the web of data.

LOD stipulates four basic principles. The first principle is that we first have to identify the items of interest in our domain. Those items are the resources, which will be described in the data. The next principle is that those resources have to be identified by HyperText Transfer Protocol $^{32}$ (HTTP) Uniform Resource Identifiers ${ }^{33}$ (URIs) and avoid schemes such as Uniform Resource Names ${ }^{34}$ (URNs) and Digital Object Identifiers ${ }^{35}$ (DOIs). The third principle is to provide useful information when accessing an HTTP URI. The fourth rule is to provide links to the outside world, i.e. to connect the data into the web of data. In practice, this means that every resource described by an RDF schema has to be identified by an HTTP URI, e.g., "http://dbpedia.org/resource/Ghent". Every resource should also have two representations: an $\mathrm{XHTML}^{36}$ and an RDF representation. Every representation also has to be identified by an HTTP URI, both in a XHTML representation $^{37}$ and in a RDF representation ${ }^{38}$. When coming across the HTTP URI of a resource, the LOD server determines which representation should be served, based on information in the accept header of the user's client, and redirects the client to the appropriate representation using 303 redirect and content negotiation.

For publishing the events using our ontology of EventsMLG2 as LOD, we rely on the D2R Server [1]. D2R Server is a tool for publishing information from relational databases on the semantic web. The tool maps the database content into RDF and allows searching and browsing this RDF data. D2R offers three interfaces to the RDF data: a) Linked Data interface: makes the RDF data available over HTTP URIs; b) SPARQL interface: enables searching and querying the database using the SPARQL query language over the SPARQL protocol; c) HTML interface: enables access to the RDF data by mapping the RDF data to a XHTML representation, accessible for HTML Web browsers. This way, the event descriptions are published as LOD, making the descriptions available for humans and machine agents.

\footnotetext{
32 http://www. ietf.org/rfc/rfc2616.txt

33 http://tools.ietf.org/html/rfc3986

34 http://www.ietf.org/rfc/rfc2141.txt

35 http://www.doi.org/

36 http://www.w3.org/TR/xhtml1/

37 http://dbpedia.org/page/Ghent

38 http://dbpedia.org/data/Ghent
} 
This promotes the reuse of the event descriptions by other applications. The recommendation engine (see Section 7) uses these descriptions to calculate recommendations, and the Facebook application (see Section 8) imports these descriptions as Facebook events using the service bus (see Section 3).

\subsection{Facebook as initial distribution platform}

By now we have built a whole events recommender system, but the system still lacks a distribution platform. The Facebook platform was chosen as one of these distribution platforms. Our recommender system uses a hybrid collaborative filtering - content based technique, as seen in Section 7 . This means that the recommendation system uses both the EventsML-G2 descriptions of the events and the profile descriptions of the users. These profile descriptions from certain user communities, grow as the users interact with the event recommendations. It is obvious that the more popular the user community is, the more user interactions can be recorded and hence, the more accurate the recommendations will become. This is the main reason why the Facebook platform was chosen as one of the distribution platforms. Another reason is that Facebook has opened up its profile and via a Facebook connect these profile data get imported in the global profile used by the recommendation system.

For the distribution on the Facebook platform, a Facebook service was developed (see Section 6). The service contacts the recommendation system for offering events to the Facebook user. The recommended events also need to be described in the Facebook platform. For this, the application imports regularly the events via the SPARQL endpoint of the events LOD server using the enterprise service bus as seen in Section 3. In this service bus the EventsML-G2 descriptions of the events are mapped to a Facebook description of events. These events can then be imported into the Facebook platform. From now on, the users can interact with these events, e.g., adding RSVP feedback, and the events can be recommended to the Facebook users. The user is also able to filter the recommendations, e.g., restricting the geographical area of the recommended events. Another distribution mechanism used for this events recommendation system is a widget, which can be used by the cultural temples on their own website. This widget contacts the recommendation system to offer personalised recommendations. Filters can be added to restrict the recommendations, e.g., to the events occurring in that cultural temple. A Facebook connect is needed here to identify the users uniquely. If the cultural temple has its own user community, these profile data can also be imported to the user's global profile.

\section{CONCLUSIONS}

In this paper, we have presented an OWL ontology for the EventsML-G2 standard as a unifying (meta)datamodel dealing with dynamic distributed cultural activities information. Using that ontology as a data communication interface within CUPID's bus architecture, several services (aggregation, categorisation, enrichment, profiling, recommendation, and distribution) were hooked in our workflow engine giving the Flemish cultural scene for the first time a tool to recommend cross-genre cultural activities, thus trying to augment customer satisfaction of the interested communities. At the same time, we provided the (inter)national cultural com- munity with mechanisms to describe and exchange event and profile information in a standardised way. We used Facebook to demonstrate the concepts of data portability of user profiles, and to generate recommendations based on a global profile, within which we integrated information fields from different social networks. We envisioned an architecture where Facebook is not the proprietary hub though. The Data Portability project ${ }^{39}$ provides promising technology tools to connect user data from a plethora of (social network) sources. Implementing our ideas with open standards like openID ${ }^{40}$ and oAuth ${ }^{41}$ is the obvious next step in our research and development.

\section{ACKNOWLEDGMENTS}

The research activities that have been described in this paper were funded by Ghent University, K.U. Leuven, VRTmedialab, Interdisciplinary Institute for Broadband Technology (IBBT) through the CUPID-project ( $50 \%$ co-funded by industrial partners), the Institute for the Promotion of Innovation by Science and Technology in Flanders (IWT), the Fund for Scientific Research-Flanders (FWO-Flanders), and the European Union.

\section{REFERENCES}

[1] C. Bizer and R. Cyganiak. D2r server - publishing releational databases on the semantic web. In Proceedings of the 5th International Semantic Web Conference, 2006.

[2] C. Cornelis, X. Guo, J. Lu, and G. Zhang. A fuzzy relational approach to event recommendation. In IICAI, pages 2231-2242, 2005.

[3] G. Karypis. Evaluation of item-based top-n recommendation algorithms. In CIKM '01: Proceedings of the tenth international conference on Information and knowledge management, pages 247-254, New York, NY, USA, 2001. ACM.

[4] K. Kurapati, S. Gutta, D. Schaffer, J. Martino, and J. Zimmerman. A multi-agent tv recommender. In Proceedings of the UM 2001 workshop Personalization in Future TV, pages 13-14, 2001.

[5] G. Linden, B. Smith, and J. York. Amazon.com recommendations: item-to-item collaborative filtering. Internet Computing, IEEE, 7(1):76-80, 2003.

[6] P. Mechant and K. Michiels. Het virtuele kunstencentrum van de toekomst. Zoektocht naar een innovatief webplatform voor virtuele cultuurbeleving. Lulu - IBBT, London - Ghent, 2007.

[7] L. H. Ungar, D. P. Foster, E. Andre, S. Wars, F. S. Wars, D. S. Wars, and J. H. Whispers. Clustering methods for collaborative filtering. In Proceedings of the Workshop on Recommendation Systems. AAAI Press, 1998.

[8] Z. Yu and X. Zhou. Tv3p: an adaptive assistant for personalized tv. Consumer Electronics, IEEE Transactions on, 50(1):393-399, Feb 2004.

[9] H. Zhang, S. Zheng, and J. Yuan. A personalized tv guide system compliant with mhp. Consumer Electronics, IEEE Transactions on, 51(2):731-737, May 2005.

\footnotetext{
39 http://www.dataportability.org/

40 http://openid.net/

41 http://openid.net/
} 